\title{
Asymptotic Stability of Impulsive Reaction-Diffusion Cellular Neural Networks with Time-Varying Delays
}

\author{
Yutian Zhang \\ College of Mathematics \& Physics, Nanjing University of Information Science E Technology, \\ Nanjing 21004, China \\ Correspondence should be addressed to Yutian Zhang, ytzhang81@163.com
}

Received 27 June 2011; Accepted 13 September 2011

Academic Editor: E. S. Van Vleck

Copyright (C) 2012 Yutian Zhang. This is an open access article distributed under the Creative Commons Attribution License, which permits unrestricted use, distribution, and reproduction in any medium, provided the original work is properly cited.

\begin{abstract}
This work addresses the asymptotic stability for a class of impulsive cellular neural networks with time-varying delays and reaction-diffusion. By using the impulsive integral inequality of Gronwall-Bellman type and Hardy-Sobolev inequality as well as piecewise continuous Lyapunov functions, we summarize some new and concise sufficient conditions ensuring the global exponential asymptotic stability of the equilibrium point. The provided stability criteria are applicable to Dirichlet boundary condition and showed to be dependent on all of the reactiondiffusion coefficients, the dimension of the space, the delay, and the boundary of the spatial variables. Two examples are finally illustrated to demonstrate the effectiveness of our obtained results.
\end{abstract}

\section{Introduction}

Cellular neural networks (CNNs), proposed by Chua and Yang in 1988 [1, 2], have been the focus of a number of investigations due to their potential applications in various fields such as optimization, linear and nonlinear programming, associative memory, pattern recognition, and computer vision [3-7]. Moreover, on the ground that time delays are unavoidably encountered for the finite switching speed of neurons and amplifiers in implementation of neural networks, it was followed by the introduction of the delayed cellular neural networks (DCNNs) so as to solve some dynamic image processing and pattern recognition problems. Such applications concerning CNNs and DCNNs depend heavily on the dynamical behaviors such as stability, convergence, and oscillatory $[8,9]$. Particularly, stability analysis has been a major concern in the designs and applications of the CNNs and DCNNs. The stability of CNNs and DCNNs is a subject of current interest, and considerable theoretical efforts have been put into this topic with many good results reported (see, e.g., [10-13]). 
With reference to neural networks, however, it is noteworthy that the state of electronic networks is actually subject to instantaneous perturbations more often than not. On this account, the networks experience abrupt change at certain instants which may be caused by a switching phenomenon, frequency change, or other sudden noise; that is, the networks often exhibit impulsive effects [14, 15]. For instance, according to Arbib [16] and Haykin [17], when a stimulus from the body or the external environment is received by receptors, the electrical impulses will be conveyed to the neural net and impulsive effects arise naturally in the net. As a consequence, in the past few years, scientists have become increasingly interested in the influence that impulses may have on the CNNs and DCNNs and a large number of stability criteria have been derived (see, e.g. [18-22]).

In reality, besides impulsive effects, diffusion effects are also nonignorable since diffusion is unavoidable when electrons are moving in asymmetric electromagnetic fields. As such, the model of neural networks with both impulses and reaction-diffusion should be more accurate to describe the evolutionary process of the systems in question, and it is necessary to consider the effects of both diffusion and impulses on the stability of CNNs and DCNNs.

In the past years, there have been a few theoretical contributions to the stability of CNNs and DCNNs with impulses and diffusion. For instance, Qiu [23] formulated a mathematical model of impulsive neural networks with time-varying delays and reactiondiffusion terms described by impulsive partial differential equations and studied, via delay impulsive differential inequality, the problem of global exponential stability with some stability criteria presented. Remarkably, all of the obtained stability criteria in [23] are independent of the diffusion. In 2008, $\mathrm{Li}$ and song [24] investigated a class of impulsive Cohen-Grossberg networks with time-varying delays and reaction-diffusion terms. By establishing a delay inequality with impulsive initial conditions and M-matrix theory, some sufficient conditions ensuring global exponential stability of the equilibrium points are given. Analogous to [23], the proposed stability criteria in [24] are also independent of the diffusion. More recently Pan et al. [25] investigated a class of impulsive Cohen-Grossberg neural networks with time-varying delays and reaction-diffusion in 2010. By the aid of the delay impulsive differential inequality quoted in [23], several sufficient conditions are exploited ensuring global exponential stability of the equilibrium points. Especially, different from $[23,24]$, the estimate of the exponential convergence rate depends on reaction-diffusion in [25].

In this paper, unlike the methods of impulsive differential inequalities and Poincare inequality used in [25], we attempt to adopt the new techniques of the impulsive integral inequality of Gronwall-Bellman type and Hardy-Sobolev inequality to investigate the problem of global exponential asymptotic stability for impulsive cellular neural networks with time-varying delays and reaction-diffusion terms. Different from the existing research, we find, besides the reaction-diffusion coefficients, the dimension of the space and the boundary of the spatial variables do influence the stability.

The rest of the paper is organized as follows. In Section 2, the model of impulsive delayed cellular neural networks with reaction-diffusion terms and Dirichlet boundary condition is outlined, and some facts and lemmas are introduced for later reference. By the new agency of the impulsive integral inequality of Gronwall-Bellman type as well as HardySobolev inequality, we discuss the global exponential asymptotic stability and develop some new criteria in Section 3. To conclude, two illustrative examples are given to verify the effectiveness of our results in Section 4. 


\section{Preliminaries}

Let $R^{n}$ denote the $n$-dimensional Euclidean space, and $\Omega \subset R^{m}$ is a bounded open set containing the origin. The boundary of $\Omega$ is smooth and mes $\Omega>0$. Let $R_{+}=[0, \infty)$ and $t_{0} \in R_{+}$.

We consider the following impulsive neural networks with time delays and reactiondiffusion terms:

$$
\begin{gathered}
\frac{\partial u_{i}(t, x)}{\partial t}=\sum_{s=1}^{m} \frac{\partial}{\partial x_{s}}\left(D_{i s} \frac{\partial u_{i}(t, x)}{\partial x_{s}}\right)-a_{i} u_{i}(t, x)+\sum_{j=1}^{n} b_{i j} f_{j}\left(u_{j}(t, x)\right)+\sum_{j=1}^{n} c_{i j} f_{j}\left(u_{j}\left(t-\tau_{j}(t), x\right)\right) \\
t \geq t_{0}, \quad t \neq t_{k}, \quad x \in \Omega, \quad i=1,2, \ldots, n, \quad k=1,2, \ldots
\end{gathered}
$$

where $n$ corresponds to the numbers of units in a neural network; $x=\left(x_{1}, \ldots, x_{m}\right)^{\mathrm{T}} \in \Omega$, $u_{i}(t, x)$, denotes the state of the $i$ th neuron at time $t$ and in space $x$; smooth functions $D_{i s}=$ $D_{i s}(t, x, u) \geq 0$ represent transmission diffusion operators of the $i$ th unit; activation functions $f_{j}\left(u_{j}(t, x)\right)$ stand for the output of the $j$ th unit at time $t$ and in space $x ; b_{i j}, c_{i j}, a_{i}$ are constants: $b_{i j}$ indicates the strength of the $j$ th unit on the $i$ th unit at time $t$ and in space $x, c_{i j}$ denotes the strength of the $j$ th unit on the $i$ th unit at time $t-\tau_{j}(t)$ and in space $x$, where $\tau_{j}(t)$ corresponds to the transmission delay along the axon of the $j$ th unit and satisfies $0 \leq \tau_{j}(t) \leq \tau$ ( $\tau=$ const) as well as $\dot{\tau}_{j}(t)<1-1 / h(h>0)$, and $a_{i}>0$ represents the rate with which the $i$ th unit will reset its potential to the resting state in isolation when disconnected from the network and external inputs at time $t$ and in space $x$. The fixed moments $t_{k}(k=1,2, \ldots)$ are called impulsive moments satisfying $0 \leq t_{0}<t_{1}<t_{2}<\cdots$ and $\lim _{k \rightarrow \infty} t_{k}=\infty ; u_{i}\left(t_{k}+0, x\right)$ and $u_{i}\left(t_{k}-0, x\right)$ represent the right-hand and left-hand limit of $u_{i}(t, x)$ at time $t_{k}$ and in space $x$, respectively. $P_{i k}\left(u_{i}\left(t_{k}, x\right)\right)$ stands for the abrupt change of $u_{i}(t, x)$ at impulsive moment $t_{k}$ and in space $x$.

Denote by $u(t, x)=u\left(t, x ; t_{0}, \varphi\right), u \in R^{n}$ the solution of system (2.1)-(2.2), satisfying the initial condition

$$
u\left(s, x ; t_{0}, \varphi\right)=\varphi(s, x), \quad t_{0}-\tau \leq s \leq t_{0}, x \in \Omega
$$

and Dirichlet boundary condition

$$
u\left(t, x ; t_{0}, \varphi\right)=0, \quad t \geq t_{0}, x \in \partial \Omega,
$$

where the vector-valued function $\varphi(s, x)=\left(\varphi_{1}(s, x), \ldots, \varphi_{n}(s, x)\right)^{T}$ is such that $\int_{\Omega} \sum_{i=1}^{n}$ $\varphi_{i}^{2}(s, x) d x$ is bounded on $\left[t_{0}-\tau, t_{0}\right]$ and $\varphi_{i}(s, x)(i=1,2, \ldots, n)$ is first-order continuous differentiable as to $s$ on $\left[t_{0}-\tau, t_{0}\right]$.

The solution $u(t, x)=u\left(t, x ; t_{0}, \varphi\right)=\left(u_{1}\left(t, x ; t_{0}, \varphi\right), \ldots, u_{n}\left(t, x ; t_{0}, \varphi\right)\right)^{T}$ of problems $((2.5)-(2.8))$ is, for the time variable $t$, a piecewise continuous function with the first kind 
discontinuity at the points $t_{k}(k=1,2, \ldots)$, where it is continuous from the left, that is the following relations are true:

$$
u_{i}\left(t_{k}-0, x\right)=u_{i}\left(t_{k}, x\right), \quad u_{i}\left(t_{k}+0, x\right)=u_{i}\left(t_{k}, x\right)+P_{i k}\left(u_{i}\left(t_{k}, x\right)\right)
$$

Throughout this paper, the norm of $u\left(t, x ; t_{0}, \varphi\right)$ is governed by

$$
\left\|u\left(t, x ; t_{0}, \varphi\right)\right\|_{\Omega}=\left(\sum_{i=1}^{n} \int_{\Omega} u_{i}^{2}\left(t, x ; t_{0}, \varphi\right) \mathrm{d} x\right)^{1 / 2}
$$

Before moving on, we introduce two hypotheses as follows.

(H1) Activation function $f_{j}\left(u_{j}(t, x)\right)$ satisfies $f_{i}(0)=0$, and there exists constant $l_{i}>0$ such that $\left|f_{i}\left(y_{1}\right)-f_{i}\left(y_{2}\right)\right| \leq l_{i}\left|y_{1}-y_{2}\right|$ holds for all $y_{1}, y_{2} \in R$ and $i=1,2, \ldots, n$.

(H2) The functions $P_{i k}\left(u_{i}\left(t_{k}, x\right)\right)$ are continuous on $R$ and $P_{i k}(0)=0, i=1,2, \ldots, n, k=$ $1,2, \ldots$

According to (H1) and (H2), it is easy to see that problems ((2.5)-(2.8)) admits an equilibrium point $u=0$.

Definition 2.1. The equilibrium point $u=0$ of problems $((2.5)-(2.8))$ is said to be globally exponentially stable if there exist constants $\kappa>0$ and $M \geq 1$ such that

$$
\left\|u\left(t, x ; t_{0}, \varphi\right)\right\|_{\Omega} \leq M \overline{\|\varphi\|_{\Omega}} e^{-\kappa\left(t-t_{0}\right)}, \quad t \geq t_{0}
$$

where ${\overline{\|\varphi\|_{\Omega}^{2}}}_{\Omega}^{2} \sup _{t_{0}-\tau \leq s \leq t_{0}} \sum_{i=1}^{n} \int_{\Omega} \varphi_{i}^{2}(s, x) \mathrm{d} x$.

Lemma 2.2 (Gronwall-Bellman-type impulsive integral inequality [26]). Assume that

(A1) the sequence $\left\{t_{k}\right\}$ satisfies $0 \leq t_{0}<t_{1}<t_{2}<\cdots$, with $\lim _{k \rightarrow \infty} t_{k}=\infty$,

(A2) $q \in P C^{1}\left[R_{+}, R\right]$ and $q(t)$ is left-continuous at $t_{k}, k=1,2, \ldots$,

(A3) $p \in C\left[R_{+}, R_{+}\right]$and for $k=1,2, \ldots$

$$
q(t) \leq c+\int_{t_{0}}^{t} p(s) q(s) d s+\sum_{t_{0}<t_{k}<t} \eta_{k} q\left(t_{k}\right), \quad t \geq t_{0},
$$

where $\eta_{k} \geq 0$ and $c=$ const. Then,

$$
q(t) \leq c \prod_{t_{0}<t_{k}<t}\left(1+\eta_{k}\right) \exp \left(\int_{t_{0}}^{t} p(s) d s\right), \quad t \geq t_{0} .
$$


Lemma 2.3 (Hardy-Sobolev inequality [27]). Let $\Omega \subset R^{m}(m \geq 3)$ be a bounded open set containing the origin and $u \in H^{1}(\Omega)=\left\{\omega \mid \omega \in L^{2}(\Omega), D_{i} \omega=\partial \omega / \partial x_{i} \in L^{2}(\Omega), 1 \leq i \leq m\right\}$. Then there exists a positive constant $C_{m}=C_{m}(\Omega)$ such that

$$
\frac{(m-2)^{2}}{4} \int_{\Omega} \frac{u^{2}}{|x|^{2}} d x \leq \int_{\Omega}|\nabla u|^{2} d x+C_{m} \int_{\partial \Omega} u^{2} d \sigma
$$

Lemma 2.4. If $a>0$ and $b>0$, then $a b \leq(1 / \varepsilon) a^{2}+\varepsilon b^{2}$ holds for any $\varepsilon>0$.

\section{Main Results}

Theorem 3.1. Provided that

(1) for $x=\left(x_{1}, \ldots, x_{m}\right)^{T} \in \Omega(m \geq 3)$, there exists a constant $\beta$ such that $|x|^{2}=\sum_{s=1}^{m} x_{s}^{2}<\beta$. In addition, there exists a constant $\underline{D}>0$ such that $D_{i s}=D_{i s}(t, x, u) \geq \underline{D}>0$. Denote $\underline{D}(m-2)^{2} / 2 \beta=x$,

(2) $P_{i k}\left(u_{i}\left(t_{k}, x\right)\right)=-\theta_{i k} u_{i}\left(t_{k}, x\right), 0 \leq \theta_{i k} \leq 2$,

(3) there exists a constant $\gamma$ satisfying $\gamma+\lambda+h \rho e^{\gamma \tau}>0$ as well as $\lambda+h \rho e^{\gamma \tau}<0$, where $\lambda=\max _{i=1, \ldots, n}\left(-\mathcal{X}-2 a_{i}+\sum_{j=1}^{n}\left(b_{i j}^{2}+c_{i j}^{2}\right)\right)+\rho, \rho=n \max _{i=1, \ldots, n}\left(l_{i}^{2}\right)$,

then, the equilibrium point $u=0$ of problems ((2.5)-(2.8)) is globally exponentially stable with convergence rate $-\left(\lambda+h \rho e^{r \tau}\right) / 2$.

Proof. Multiplying both sides of $(2.1)$ by $u_{i}(t, x)$ and integrating with respect to spatial variable $x$ on $\Omega$, we get

$$
\begin{aligned}
\frac{\mathrm{d}\left(\int_{\Omega} u_{i}^{2}(t, x) \mathrm{d} x\right)}{\mathrm{d} t}= & 2 \sum_{s=1}^{m} \int_{\Omega} u_{i}(t, x) \frac{\partial}{\partial x_{s}}\left(D_{i s} \frac{\partial u_{i}(t, x)}{\partial x_{s}}\right) \mathrm{d} x-2 a_{i} \int_{\Omega} u_{i}^{2}(t, x) \mathrm{d} x \\
& +2 \sum_{j=1}^{n} b_{i j} \int_{\Omega} u_{i}(t, x) f_{j}\left(u_{j}(t, x)\right) \mathrm{d} x \\
& +2 \sum_{j=1}^{n} c_{i j} \int_{\Omega} u_{i}(t, x) f_{j}\left(u_{j}\left(t-\tau_{j}(t), x\right)\right) \mathrm{d} x \quad t \geq t_{0}, t \neq t_{k}, k=1,2, \ldots
\end{aligned}
$$

Regarding the right-hand part of (3.1), the first term becomes by using Green formula, Dirichlet boundary condition, Lemma 2.3, and condition 1 of Theorem 3.1

$$
\begin{aligned}
& 2 \sum_{s=1}^{m} \int_{\Omega} u_{i}(t, x) \frac{\partial}{\partial x_{s}}\left(D_{i s} \frac{\partial u_{i}(t, x)}{\partial x_{s}}\right) \mathrm{d} x=-2 \sum_{s=1}^{m} \int_{\Omega} D_{i s}\left(\frac{\partial u_{i}(t, x)}{\partial x_{s}}\right)^{2} \mathrm{~d} x \\
& \leq-\frac{\underline{D}(m-2)^{2}}{2} \int_{\Omega} \frac{u_{i}^{2}(t, x)}{|x|^{2}} d x \leq-\frac{D}{(m-2)^{2}} \int_{\Omega} u_{i}^{2}(t, x) d x \triangleq-x \int_{\Omega} u_{i}^{2}(t, x) \mathrm{d} x .
\end{aligned}
$$


Moreover, we derive from (H1) that

$$
\begin{aligned}
2 \sum_{j=1}^{n} b_{i j} \int_{\Omega} u_{i}(t, x) f_{j}\left(u_{j}(t, x)\right) \mathrm{d} x & \leq 2 \sum_{j=1}^{n}\left|b_{i j}\right| \int_{\Omega}\left|u_{i}(t, x)\right|\left|f_{j}\left(u_{j}(t, x)\right)\right| \mathrm{d} x \\
& \leq 2 \sum_{j=1}^{n} \int_{\Omega} l_{j}\left|b_{i j}\right|\left|u_{i}(t, x)\right|\left|u_{j}(t, x)\right| \mathrm{d} x \\
& \leq \sum_{j=1}^{n} \int_{\Omega}\left(b_{i j}^{2} u_{i}^{2}(t, x)+l_{j}^{2} u_{i}^{2}(t, x)\right) \mathrm{d} x, \\
2 \sum_{j=1}^{n} c_{i j} \int_{\Omega} u_{i}(t, x) f_{j}\left(u_{j}\left(t-\tau_{j}(t), x\right)\right) d x & \leq 2 \sum_{j=1}^{n}\left|c_{i j}\right| \int_{\Omega}\left|u_{i}(t, x)\right|\left|f_{j}\left(u_{j}\left(t-\tau_{j}(t), x\right)\right)\right| d x \\
& \leq 2 \sum_{j=1}^{n} \int_{\Omega} l_{j}\left|c_{i j}\right|\left|u_{i}(t, x)\right|\left|u_{j}\left(t-\tau_{j}(t), x\right)\right| d x \\
& \leq \sum_{j=1}^{n} \int_{\Omega}\left(c_{i j}^{2} u_{i}^{2}(t, x)+l_{j}^{2} u_{i}^{2}\left(t-\tau_{j}(t), x\right)\right) d x .
\end{aligned}
$$

Consequently, substituting ((2.10)-(3.14)) into (3.1) produces

$$
\begin{aligned}
\frac{\mathrm{d}\left(\int_{\Omega} u_{i}^{2}(t, x) \mathrm{d} x\right)}{\mathrm{d} t} \leq & -x \int_{\Omega} u_{i}^{2}(t, x) \mathrm{d} x-2 a_{i} \int_{\Omega} u_{i}^{2}(t, x) \mathrm{d} x \\
& +\sum_{j=1}^{n} \int_{\Omega}\left(b_{i j}^{2} u_{i}^{2}(t, x)+l_{j}^{2} u_{i}^{2}(t, x)\right) \mathrm{d} x \\
& +\sum_{j=1}^{n} \int_{\Omega}\left(c_{i j}^{2} u_{i}^{2}(t, x)+l_{j}^{2} u_{i}^{2}\left(t-\tau_{j}(t), x\right)\right) \mathrm{d} x
\end{aligned}
$$

for $t \geq t_{0}, t \neq t_{k}, k=1,2, \ldots$

We define a Lyapunov function $V_{i}(t)$ as $V_{i}(t)=\int_{\Omega} u_{i}^{2}(t, x) \mathrm{d} x$. It is easy to find that $V_{i}(t)$ is a piecewise continuous function with points of discontinuity of the first kind $t_{k}(k=$ $1,2, \ldots)$, where it is continuous from the left, that is, $V_{i}\left(t_{k}-0\right)=V_{i}\left(t_{k}\right)(k=1,2, \ldots)$. In addition, due to $V_{i}\left(t_{0}+0\right) \leq V_{i}\left(t_{0}\right)$ and the following estimate derived from condition 2 of Theorem 3.1

$$
u_{i}^{2}\left(t_{k}+0, x\right)=\left(-\theta_{i k} u_{i}\left(t_{k}, x\right)+u_{i}\left(t_{k}, x\right)\right)^{2}=\left(1-\theta_{i k}\right)^{2} u_{i}^{2}\left(t_{k}, x\right) \leq u_{i}^{2}\left(t_{k}, x\right) \quad(k=1,2, \ldots),
$$

we have

$$
V_{i}\left(t_{k}+0\right) \leq V_{i}\left(t_{k}\right), \quad k=0,1,2, \ldots
$$


holds for $t=t_{k}(k=0,1,2, \ldots)$. Put $t \in\left(t_{k}, t_{k+1}\right), k=0,1,2, \ldots$ Then for the derivative $\mathrm{d} V_{i}(t) / \mathrm{d} t$ of $V_{i}$ with respect to problems ((2.5)-(2.8)), it results from (3.4) that

$$
\begin{aligned}
\frac{\mathrm{d} V_{i}(t)}{\mathrm{d} t} \leq & -x \int_{\Omega} u_{i}^{2}(t, x) \mathrm{d} x-2 a_{i} \int_{\Omega} u_{i}^{2}(t, x) \mathrm{d} x+\sum_{j=1}^{n} \int_{\Omega}\left(b_{i j}^{2} u_{i}^{2}(t, x)+l_{j}^{2} u_{i}^{2}(t, x)\right) \mathrm{d} x \\
& +\sum_{j=1}^{n} \int_{\Omega}\left(c_{i j}^{2} u_{i}^{2}(t, x)+l_{j}^{2} u_{j}^{2}\left(t-\tau_{j}(t), x\right)\right) \mathrm{d} x \leq\left(-x-2 a_{i}+\sum_{j=1}^{n} b_{i j}^{2}+\sum_{j=1}^{n} c_{i j}^{2}\right) V_{i}(t) \\
& +\max _{i=1, \ldots, n}\left(l_{i}^{2}\right) \sum_{j=1}^{n} V_{j}(t)+\max _{i=1, \ldots, n}\left(l_{i}^{2}\right) \sum_{j=1}^{n} V_{j}\left(t-\tau_{j}(t)\right) \quad t \in\left(t_{k}, t_{k+1}\right), k=0,1,2, \ldots
\end{aligned}
$$

Choose $V(t)$ of the form $V(t)=\sum_{i=1}^{n} V_{i}(t)$. From (3.7), one then reads

$$
\begin{aligned}
\frac{\mathrm{d} V(t)}{\mathrm{d} t} \leq & \left(\max _{i=1, \ldots, n}\left(-x-2 a_{i}+\sum_{j=1}^{n}\left(b_{i j}^{2}+c_{i j}^{2}\right)\right)+n \max _{i=1, \ldots, n}\left(l_{i}^{2}\right)\right) V(t) \\
& +n \max _{i=1, \ldots, n}\left(l_{i}^{2}\right) \sum_{j=1}^{n} V_{j}\left(t-\tau_{j}\right) \\
= & \lambda V(t)+\rho \sum_{j=1}^{n} V_{j}\left(t-\tau_{j}(t)\right) \quad t \in\left(t_{k}, t_{k+1}\right), k=0,1,2, \ldots
\end{aligned}
$$

Construct $V^{*}(t)=\mathrm{e}^{\gamma\left(t-t_{0}\right)} V(t)$, where $\gamma$ satisfies $\gamma+\lambda+h \rho e^{\gamma \tau}>0$ and $\lambda+h \rho e^{\gamma \tau}<0$. Evidently, $V^{*}(t)$ is also a piecewise continuous function with points of discontinuity of the first kind $t_{k}(k=1,2, \ldots)$, in which it is continuous from the left, that is $V^{*}\left(t_{k}-0\right)=V^{*}\left(t_{k}\right)$ $(k=1,2, \ldots)$. Moreover, at $t=t_{k}(k=0,1,2, \ldots)$, we find by use of (3.6)

$$
V^{*}\left(t_{k}+0\right) \leq V^{*}\left(t_{k}\right), \quad k=0,1,2, \ldots
$$

Set $t \in\left(t_{k}, t_{k+1}\right), k=0,1,2, \ldots$ By virtue of (3.8), one has

$$
\begin{aligned}
\frac{\mathrm{d} V^{*}(t)}{\mathrm{d} t} & =\gamma \mathrm{e}^{\gamma\left(t-t_{0}\right)} V(t)+\mathrm{e}^{\gamma\left(t-t_{0}\right)} \frac{\mathrm{d} V(t)}{\mathrm{d} t} \leq \gamma \mathrm{e}^{\gamma\left(t-t_{0}\right)} V(t)+\left(\lambda V(t)+\rho \sum_{j=1}^{n} V_{j}\left(t-\tau_{j}(t)\right)\right) \mathrm{e}^{\gamma\left(t-t_{0}\right)} \\
& =(\gamma+\lambda) V^{*}(t)+\rho \mathrm{e}^{\gamma\left(t-t_{0}\right)} \sum_{j=1}^{n} V_{j}\left(t-\tau_{j}(t)\right) \quad t \in\left(t_{k}, t_{k+1}\right), k=0,1,2, \ldots
\end{aligned}
$$


Choose small enough $\varepsilon>0$. Integrating (3.10) from $t_{k}+\varepsilon$ to $t$ gives

$$
\begin{aligned}
V^{*}(t) \leq & V^{*}\left(t_{k}+\varepsilon\right)+(\gamma+\lambda) \int_{t_{k}+\varepsilon}^{t} V^{*}(s) d s \\
& +\int_{t_{k}+\varepsilon}^{t} \rho \mathrm{e}^{\gamma\left(s-t_{0}\right)} \sum_{j=1}^{n} V_{j}\left(s-\tau_{j}(s)\right) d s, \quad t \in\left(t_{k}, t_{k+1}\right), k=0,1,2, \ldots
\end{aligned}
$$

which yields after letting $\varepsilon \rightarrow 0$ in (3.11)

$$
\begin{aligned}
V^{*}(t) \leq & V^{*}\left(t_{k}+0\right)+(\gamma+\lambda) \int_{t_{k}}^{t} V^{*}(s) d s \\
& +\int_{t_{k}}^{t} \rho \mathrm{e}^{\gamma\left(s-t_{0}\right)} \sum_{j=1}^{n} V_{j}\left(s-\tau_{j}(s)\right) d s, \quad t \in\left(t_{k}, t_{k+1}\right), k=0,1,2, \ldots
\end{aligned}
$$

We now proceed to estimate the value of $V^{*}(t)$ at $t=t_{k+1}, k=0,1,2, \ldots$. For small enough $\varepsilon>0$, we put $t=t_{k+1}-\varepsilon$. Now an application of (3.12) leads to, for $k=0,1,2, \ldots$,

$$
V^{*}\left(t_{k+1}-\varepsilon\right) \leq V^{*}\left(t_{k}+0\right)+(\gamma+\lambda) \int_{t_{k}}^{t_{k+1}-\varepsilon} V^{*}(s) d s+\int_{t_{k}}^{t_{k+1}-\varepsilon} \rho \mathrm{e}^{\gamma\left(s-t_{0}\right)} \sum_{j=1}^{n} V_{j}\left(s-\tau_{j}(s)\right) d s .
$$

If we let $\varepsilon \rightarrow 0$ in (3.13), there results

$$
\begin{aligned}
V^{*}\left(t_{k+1}-0\right) \leq & V^{*}\left(t_{k}+0\right)+(\gamma+\lambda) \int_{t_{k}}^{t_{k+1}} V^{*}(s) d s \\
& +\int_{t_{k}}^{t_{k+1}} \rho \mathrm{e}^{\gamma\left(s-t_{0}\right)} \sum_{j=1}^{n} V_{j}\left(s-\tau_{j}(s)\right) d s, \quad k=0,1,2, \ldots
\end{aligned}
$$

Note that $V^{*}\left(t_{k+1}-0\right)=V^{*}\left(t_{k+1}\right)$ is applicable for $k=0,1,2, \ldots$ Thus,

$$
V^{*}\left(t_{k+1}\right) \leq V^{*}\left(t_{k}+0\right)+(\gamma+\lambda) \int_{t_{k}}^{t_{k+1}} V^{*}(s) d s+\int_{t_{k}}^{t_{k+1}} \rho \mathrm{e}^{\gamma\left(s-t_{0}\right)} \sum_{j=1}^{n} V_{j}\left(s-\tau_{j}(s)\right) d s
$$

holds for $k=0,1,2, \ldots$ By synthesizing (3.12) and (3.15), we then arrive at

$$
\begin{aligned}
V^{*}(t) \leq & V^{*}\left(t_{k}+0\right)+(\gamma+\lambda) \int_{t_{k}}^{t} V^{*}(s) d s \\
& +\int_{t_{k}}^{t} \rho \mathrm{e}^{\gamma\left(s-t_{0}\right)} \sum_{j=1}^{n} V_{j}\left(s-\tau_{j}(s)\right) d s \quad t \in\left(t_{k}, t_{k+1}\right], k=0,1,2, \ldots
\end{aligned}
$$


Journal of Applied Mathematics

This, together with (3.9), results in

$$
V^{*}(t) \leq V^{*}\left(t_{k}\right)+(\gamma+\lambda) \int_{t_{k}}^{t} V^{*}(s) d s+\int_{t_{k}}^{t} \rho \mathrm{e}^{\gamma\left(s-t_{0}\right)} \sum_{j=1}^{n} V_{j}\left(s-\tau_{j}(s)\right) d s
$$

for $t \in\left(t_{k}, t_{k+1}\right], k=0,1,2, \ldots$

Recalling assumptions that $0 \leq \tau_{j}(t) \leq \tau$ and $\dot{\tau}_{j}(t)<1-1 / h(h>0)$, we have

$$
\begin{aligned}
\int_{t_{k}}^{t} \rho \mathrm{e}^{\gamma\left(s-t_{0}\right)} \sum_{j=1}^{n} V_{j}\left(s-\tau_{j}((s))\right) d s & =\sum_{j=1}^{n} \int_{t_{k}-\tau_{j}\left(t_{k}\right)}^{t-\tau_{j}(t)} \rho \mathrm{e}^{\gamma\left(\theta+\tau_{j}(s)-t_{0}\right)} V_{j}(\theta) \frac{1}{1-\dot{\tau}_{j}(s)} d \theta \\
& \leq h \rho e^{\gamma \tau} \sum_{j=1}^{n} \int_{t_{k}-\tau_{j}\left(t_{k}\right)}^{t-\tau_{j}(t)} \mathrm{e}^{\gamma\left(\theta-t_{0}\right)} V_{j}(\theta) d \theta .
\end{aligned}
$$

Hence,

$$
\begin{array}{r}
V^{*}(t) \leq V^{*}\left(t_{k}\right)+(\gamma+\lambda) \int_{t_{k}}^{t} V^{*}(s) d s+h \rho e^{\gamma \tau} \sum_{j=1}^{n} \int_{t_{k}-\tau_{j}\left(t_{k}\right)}^{t-\tau_{j}(t)} \mathrm{e}^{\gamma\left(s-t_{0}\right)} V_{j}(s) d s \\
t \in\left(t_{k}, t_{k+1}\right], \quad k=0,1,2, \ldots
\end{array}
$$

By induction argument, we reach

$$
\begin{gathered}
V^{*}\left(t_{k}\right) \leq V^{*}\left(t_{k-1}\right)+(\gamma+\lambda) \int_{t_{k-1}}^{t_{k}} V^{*}(s) d s+h \rho e^{\gamma \tau} \sum_{j=1}^{n} \int_{t_{k-1}-\tau_{j}\left(t_{k-1}\right)}^{t_{k}-\tau_{j}\left(t_{k}\right)} \mathrm{e}^{\gamma\left(s-t_{0}\right)} V_{j}(s) d s, \\
\vdots \\
V^{*}\left(t_{2}\right) \leq V^{*}\left(t_{1}\right)+(\gamma+\lambda) \int_{t_{1}}^{t_{2}} V^{*}(s) d s+h \rho e^{\gamma \tau} \sum_{j=1}^{n} \int_{t_{1}-\tau_{j}\left(t_{1}\right)}^{t_{2}-\tau_{j}\left(t_{2}\right)} \mathrm{e}^{\gamma\left(s-t_{0}\right)} V_{j}(s) d s, \\
V^{*}\left(t_{1}\right) \leq V^{*}\left(t_{0}\right)+(\gamma+\lambda) \int_{t_{0}}^{t_{1}} V^{*}(s) d s+h \rho e^{\gamma \tau} \sum_{j=1}^{n} \int_{t_{0}-\tau_{j}\left(t_{0}\right)}^{t_{1}-\tau_{j}\left(t_{1}\right)} \mathrm{e}^{\gamma\left(s-t_{0}\right)} V_{j}(s) d s .
\end{gathered}
$$

Therefore,

$$
\begin{aligned}
V^{*}(t) & \leq V^{*}\left(t_{0}\right)+(\gamma+\lambda) \int_{t_{0}}^{t} V^{*}(s) d s+h \rho e^{\gamma \tau} \sum_{j=1}^{n} \int_{t_{0}-\tau_{j}\left(t_{0}\right)}^{t-\tau_{j}(t)} \mathrm{e}^{\gamma\left(s-t_{0}\right)} V_{j}(s) d s \\
& \leq V^{*}\left(t_{0}\right)+(\gamma+\lambda) \int_{t_{0}}^{t} V^{*}(s) d s+h \rho e^{\gamma \tau} \sum_{j=1}^{n} \int_{t_{0}-\tau_{j}\left(t_{0}\right)}^{t} \mathrm{e}^{\gamma\left(s-t_{0}\right)} V_{j}(s) d s
\end{aligned}
$$




$$
\begin{aligned}
= & V^{*}\left(t_{0}\right)+\left(\gamma+\lambda+h \rho e^{\gamma \tau}\right) \int_{t_{0}}^{t} V^{*}(s) d s \\
& +h \rho e^{r \tau} \sum_{j=1}^{n} \int_{t_{0}-\tau_{j}\left(t_{0}\right)}^{t_{0}} \mathrm{e}^{\gamma\left(s-t_{0}\right)} V_{j}(s) d s \quad t \in\left(t_{k}, t_{k+1}\right], k=0,1,2, \ldots .
\end{aligned}
$$

Since

$$
\begin{array}{r}
h \rho e^{\gamma \tau} \sum_{j=1}^{n} \int_{t_{0}-\tau_{j}\left(t_{0}\right)}^{t_{0}} \mathrm{e}^{\gamma\left(s-t_{0}\right)} V_{j}(s) d s \leq h \rho e^{\gamma \tau} \sum_{j=1}^{n} \int_{t_{0}-\tau}^{t_{0}} V_{j}(s) d s \\
=h \rho e^{\gamma \tau} \int_{t_{0}-\tau}^{t_{0}}\left(\sum_{j=1}^{n} \int_{\Omega} \varphi_{j}^{2}(s, x) d x\right) d s \leq \tau h \rho e^{\gamma \tau}{\overline{\|\varphi\|_{\Omega^{\prime}}^{2}}}^{2}
\end{array}
$$

we claim

$$
V^{*}(t) \leq V^{*}\left(t_{0}\right)+\tau h \rho e^{\gamma \tau}{\overline{\|\varphi\|_{\Omega}^{2}}}^{2}\left(\gamma+\lambda+h \rho e^{\gamma \tau}\right) \int_{t_{0}}^{t} V^{*}(s) d s \quad t \in\left(t_{k}, t_{k+1}\right], k=0,1,2 \ldots
$$

According to Lemma 2.2, we claim

$$
V^{*}(t) \leq\left(V^{*}\left(t_{0}\right)+\tau h \rho e^{r \tau}{\overline{\|\varphi\|_{\Omega}^{2}}}_{\Omega} \exp \left\{\left(\gamma+\lambda+h \rho e^{\gamma \tau}\right)\left(t-t_{0}\right)\right\}, \quad t \geq t_{0}\right.
$$

which reduces to

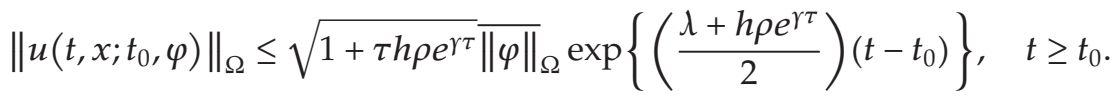

This completes the proof.

Remark 3.2. According to the conditions of Theorem 3.1, we see that the reaction-diffusion term do influence the stability of problem $((2.5)-(2.8))$. Moreover, besides the reactiondiffusion coefficients, the dimension of the space and the boundary of spatial variables have also an effect on the stability of the equilibrium point $u=0$.

Theorem 3.3. Providing that

(1) for $x=\left(x_{1}, \ldots, x_{m}\right)^{T} \in \Omega(m \geq 3)$, there exist constants $\beta$ such that $|x|^{2}=\sum_{s=1}^{m} x_{s}^{2}<\beta$, in addition, there exists constant $\underline{D}>0$ such that $D_{i s}=D_{i s}(t, x, u) \geq \underline{D}>0$; denote $\underline{D}(m-2)^{2} / 2 \beta=X$,

(2) $P_{i k}\left(u_{i}\left(t_{k}, x\right)\right)=-\theta_{i k} u_{i}\left(t_{k}, x\right), 1-\sqrt{1+\alpha} \leq \theta_{i k} \leq 1+\sqrt{1+\alpha}, \alpha \geq 0$,

(3) $\inf _{k=1,2 \ldots}\left(t_{k}-t_{k-1}\right)>\mu$, 
(4) there exists constant $\gamma$ which satisfies $\gamma+\lambda+h \rho e^{\gamma \tau}>0$ and $\lambda+h \rho e^{\gamma \tau}+\ln (1+\alpha) / \mu<0$, where $\lambda=\max _{i=1, \ldots, n}\left(-x-2 a_{i}+\sum_{j=1}^{n}\left(b_{i j}^{2}+c_{i j}^{2}\right)\right)+\rho$ and $\rho=n \max _{i=1, \ldots, n}\left(l_{i}^{2}\right)$, then, the equilibrium point $u=0$ of problem $((2.5)-(2.8))$ is globally exponentially stable with convergence rate $-(1 / 2)\left(\lambda+h \rho e^{\gamma \tau}+\ln (1+\alpha) / \mu\right)$.

Proof. Define a Lyapunov function $V$ of the form $V(t)=\sum_{i=1}^{n} V_{i}(t)$, where $V_{i}(t)=\int_{\Omega} u_{i}^{2}(t, x) \mathrm{d} x$. Obviously, $V(t)$ is a piecewise continuous function with points of discontinuity of the first kind $t_{k}, k=1,2, \ldots$, where it is continuous from the left, that is, $V_{1}\left(t_{k}-0\right)=V_{1}\left(t_{k}\right)(k=1,2, \ldots)$. Furthermore, for $t=t_{k}(k=0,1,2, \ldots)$, it follows from condition 2 of Theorem 3.3 that

$$
u_{i}^{2}\left(t_{k}+0, x\right)-u_{i}^{2}\left(t_{k}, x\right)=\left(1-\theta_{i k}\right)^{2} u_{i}^{2}\left(t_{k}, x\right)-u_{i}^{2}\left(t_{k}, x\right) \leq \alpha u_{i}^{2}\left(t_{k}, x\right)
$$

Thereby,

$$
V\left(t_{k}+0\right) \leq \alpha V\left(t_{k}\right)+V\left(t_{k}\right), k=0,1,2, \ldots
$$

Construct another Lyapunov function defined by $V^{*}(t)=\mathrm{e}^{\gamma\left(t-t_{0}\right)} V(t)$, where $\gamma$ satisfies $\gamma+\lambda+h \rho e^{\gamma \tau}>0$ and $\lambda+h \rho e^{\gamma \tau}+(\ln (1+\alpha) / \mu)<0$. Then, $V^{*}(t)$ is also a piecewise continuous function with points of discontinuity of the first kind $t_{k}, k=1,2, \ldots$, where it is continuous from the left, that is $V^{*}\left(t_{k}-0\right)=V^{*}\left(t_{k}\right)(k=1,2, \ldots)$. And for $t=t_{k}(k=0,1,2, \ldots)$, it results from (3.27) that

$$
V^{*}\left(t_{k}+0\right) \leq \alpha V^{*}\left(t_{k}\right)+V^{*}\left(t_{k}\right), \quad k=0,1,2, \ldots
$$

Set $t \in\left(t_{k}, t_{k+1}\right], k=0,1,2, \ldots$ Following the same procedure as in Theorem 3.1, we get

$$
\begin{aligned}
V^{*}(t) \leq V^{*}\left(t_{k}+0\right)+(\gamma+\lambda) & \int_{t_{k}}^{t} V^{*}(s) d s+h \rho e^{\gamma \tau} \\
& \times \sum_{j=1}^{n} \int_{t_{k}-\tau_{j}\left(t_{k}\right)}^{t-\tau_{j}(t)} \mathrm{e}^{\gamma\left(\theta-t_{0}\right)} V_{j}(\theta) d \theta \quad t \in\left(t_{k}, t_{k+1}\right], k=0,1,2, \ldots
\end{aligned}
$$

The relations (3.28) and (3.29) yield

$$
\begin{aligned}
V^{*}(t)-V^{*}\left(t_{k}\right) \leq \alpha V^{*}\left(t_{k}\right)+(\gamma+\lambda) & \int_{t_{k}}^{t} V^{*}(s) d s+h \rho e^{\gamma \tau} \\
& \times \sum_{j=1}^{n} \int_{t_{k}-\tau_{j}\left(t_{k}\right)}^{t-\tau_{j}(t)} \mathrm{e}^{\gamma\left(\theta-t_{0}\right)} V_{j}(\theta) d \theta \quad t \in\left(t_{k}, t_{k+1}\right], k=0,1,2, \ldots
\end{aligned}
$$


By induction argument, we arrive at

$$
\begin{gathered}
V^{*}\left(t_{k}\right)-V^{*}\left(t_{k-1}\right) \leq \alpha V^{*}\left(t_{k-1}\right)+(\gamma+\lambda) \int_{t_{k-1}}^{t_{k}} V^{*}(s) d s+h \rho e^{\gamma \tau} \sum_{j=1}^{n} \int_{t_{k-1}-\tau_{j}\left(t_{k-1}\right)}^{t_{k}-\tau_{j}\left(t_{k}\right)} \mathrm{e}^{\gamma\left(\theta-t_{0}\right)} V_{j}(\theta) d \theta, \\
\vdots \\
V^{*}\left(t_{2}\right)-V^{*}\left(t_{1}\right) \leq \alpha V^{*}\left(t_{1}\right)+(\gamma+\lambda) \int_{t_{1}}^{t_{2}} V^{*}(s) d s+h \rho e^{\gamma \tau} \sum_{j=1}^{n} \int_{t_{1}-\tau_{j}\left(t_{1}\right)}^{t_{2}-\tau_{j}\left(t_{2}\right)} \mathrm{e}^{\gamma\left(\theta-t_{0}\right)} V_{j}(\theta) d \theta, \\
V^{*}\left(t_{1}\right)-V^{*}\left(t_{0}\right) \leq \alpha V^{*}\left(t_{0}\right)+(\gamma+\lambda) \int_{t_{0}}^{t_{1}} V^{*}(s) d s+h \rho e^{\gamma \tau} \sum_{j=1}^{n} \int_{t_{0}-\tau_{j}\left(t_{0}\right)}^{t_{1}-\tau_{j}\left(t_{1}\right)} \mathrm{e}^{\gamma\left(\theta-t_{0}\right)} V_{j}(\theta) d \theta .
\end{gathered}
$$

Hence,

$$
\begin{aligned}
V^{*}(t)-V^{*}\left(t_{0}\right) \leq & \alpha V^{*}\left(t_{0}\right)+(\gamma+\lambda) \int_{t_{0}}^{t} V^{*}(s) d s \\
& +h \rho e^{r \tau} \sum_{j=1}^{n} \int_{t_{0}-\tau_{j}\left(t_{0}\right)}^{t-\tau_{j}(t)} \mathrm{e}^{\gamma\left(\theta-t_{0}\right)} V_{j}(\theta) d \theta+\alpha \sum_{t_{0}<t_{k}<t} V\left(t_{k}\right) \\
\leq & \alpha V^{*}\left(t_{0}\right)+\left(\gamma+\lambda+h \rho e^{\gamma \tau}\right) \int_{t_{0}}^{t} V^{*}(s) d s \\
& +h \rho e^{r \tau} \sum_{j=1}^{n} \int_{t_{0}-\tau_{j}\left(t_{0}\right)}^{t_{0}} \mathrm{e}^{\gamma\left(\theta-t_{0}\right)} V_{j}(\theta) d \theta+\alpha \sum_{t_{0}<t_{k}<t} V\left(t_{k}\right) \quad t \in\left(t_{k}, t_{k+1}\right], k=0,1,2, \ldots
\end{aligned}
$$

Introducing $h \rho e^{r \tau} \sum_{j=1}^{n} \int_{t_{0}-\tau_{j}\left(t_{0}\right)}^{t_{0}} \mathrm{e}^{\gamma\left(\theta-t_{0}\right)} V_{j}(\theta) d \theta \leq \tau h \rho e^{\gamma \tau}{\overline{\|\varphi\|_{\Omega}}}_{\Omega}^{2}$ as shown in the proof of Theorem 3.1 into (3.32), the expression becomes

$$
\begin{aligned}
V^{*}(t)-V^{*}\left(t_{0}\right) \leq & \alpha V^{*}\left(t_{0}\right)+\tau h \rho e^{\gamma \tau}{\overline{\|\varphi\|_{\Omega}^{2}}}^{2}+\left(\gamma+\lambda+h \rho e^{\gamma \tau}\right) \int_{t_{0}}^{t} V^{*}(s) d s \\
& +\alpha \sum_{t_{0}<t_{k}<t} V\left(t_{k}\right) \quad t \in\left(t_{k}, t_{k+1}\right], k=0,1,2 \ldots
\end{aligned}
$$

It then results from Lemma 2.2 that

$$
\begin{aligned}
V^{*}(t) & \leq\left((\alpha+1) V^{*}\left(t_{0}\right)+\tau h \rho e^{\gamma \tau}{\overline{\|\varphi\|_{\Omega}^{2}}}_{t_{t_{0}<t_{k}<t}}(1+\alpha) \exp \left(\left(\gamma+\lambda+h \rho e^{\gamma \tau}\right)\left(t-t_{0}\right)\right)\right. \\
& =\left((\alpha+1) V^{*}\left(t_{0}\right)+\tau h \rho e^{\gamma \tau}{\overline{\|\varphi\|_{\Omega}^{2}}}^{2}\right)(1+\alpha)^{k} \exp \left(\left(\gamma+\lambda+h \rho e^{\gamma \tau}\right)\left(t-t_{0}\right)\right), \quad t \geq t_{0} .
\end{aligned}
$$


On the other hand, since $\inf _{k=1,2, \ldots}\left(t_{k}-t_{k-1}\right)>\mu$, one has $k<\left(t_{k}-t_{0}\right) / \mu$. Thereby,

$$
(1+\alpha)^{k}<\exp \left\{\frac{\ln (1+\alpha)}{\mu}\left(t_{k}-t_{0}\right)\right\}<\exp \left\{\frac{\ln (1+\alpha)}{\mu}\left(t-t_{0}\right)\right\}
$$

And (3.34) can be rewritten as

$$
V^{*}(t) \leq\left((\alpha+1) V^{*}\left(t_{0}\right)+\tau h \rho e^{\gamma \tau}{\overline{\|\varphi\|_{\Omega}^{2}}}^{2}\right) \exp \left(\left(\gamma+\lambda+h \rho e^{\gamma \tau}+\frac{\ln (1+\alpha)}{\mu}\right)\left(t-t_{0}\right)\right)
$$

which implies

$$
\left\|u\left(t, x ; t_{0}, \varphi\right)\right\|_{\Omega} \leq \sqrt{\left(\alpha+1+\tau h \rho e^{\gamma \tau}\right)} \overline{\| \varphi}_{\Omega} \exp \left(\frac{1}{2}\left(\lambda+h \rho e^{r \tau}+\frac{\ln (1+\alpha)}{\mu}\right)\left(t-t_{0}\right)\right), \quad t \geq t_{0} .
$$

The proof is completed.

Remark 3.4. Theorem 3.1 is in fact the special case of Theorem 3.3 by choosing $\alpha=0$. Due to Lemma 2.4, we know

$$
\begin{gathered}
2 \sum_{j=1}^{n} b_{i j} \int_{\Omega} u_{i}(t, x) f\left(u_{j}(t, x)\right) \mathrm{d} x \leq \sum_{j=1}^{n} \int_{\Omega}\left(\varepsilon_{1} b_{i j}^{2} u_{i}^{2}(t, x)+\frac{l_{j}^{2}}{\varepsilon_{1}} u_{i}^{2}(t, x)\right) \mathrm{d} x, \\
2 \sum_{j=1}^{n} c_{i j} \int_{\Omega} u_{i}(t, x) f\left(u_{j}\left(t-\tau_{j}, x\right)\right) \mathrm{d} x \leq \sum_{j=1}^{n} \int_{\Omega}\left(\varepsilon_{2} c_{i j}^{2} u_{i}^{2}(t, x)+\frac{l_{j}^{2}}{\varepsilon_{2}} u_{i}^{2}\left(t-\tau_{j}, x\right)\right) \mathrm{d} x
\end{gathered}
$$

hold for any $\varepsilon_{1}, \varepsilon_{2}>0$.

In the sequel, we follow the same procedures as in Theorems 3.1 and 3.3 to find the following theorems.

\section{Theorem 3.5. Provided that}

(1) for $x=\left(x_{1}, \ldots, x_{m}\right)^{T} \in \Omega(m \geq 3)$, there exists a constant $\beta$ such that $|x|^{2}=\sum_{s=1}^{m} x_{s}^{2}<\beta$. in addition, there exists a constant $\underline{D}>0$ such that $D_{i s}=D_{i s}(t, x, u) \geq \underline{D}>0$; denote $\underline{D}(m-2)^{2} / 2 \beta=x$,

(2) $P_{i k}\left(u_{i}\left(t_{k}, x\right)\right)=-\theta_{i k} u_{i}\left(t_{k}, x\right), 0 \leq \theta_{i k} \leq 2$,

(3) there exist constants $\gamma$ and $\varepsilon_{1}, \varepsilon_{2}>0$ such that $\gamma+\lambda+h \rho e^{\gamma \tau}>0$ and $\lambda+h \rho e^{\gamma \tau}<0$, where $\lambda=\max _{i=1, \ldots, n}\left(-x-2 a_{i}+\sum_{j=1}^{n}\left(\varepsilon_{1} b_{i j}^{2}+\varepsilon_{2} c_{i j}^{2}\right)\right)+\left(n / \varepsilon_{1}\right) \max _{i=1, \ldots, n}\left(l_{i}^{2}\right)$ and $\rho=$ $\left(n / \varepsilon_{2}\right) \max _{i=1, \ldots, n}\left(l_{i}^{2}\right)$, then, the equilibrium point $u=0$ of problem $((2.5)-(2.8))$ is globally exponentially stable with convergence rate $-\left(\lambda+h \rho e^{r \tau}\right) / 2$.

Theorem 3.6. Assume that

(1) for $x=\left(x_{1}, \ldots, x_{m}\right)^{T} \in \Omega(m \geq 3)$, there exists a constant $\beta$ such that $|x|^{2}=\sum_{s=1}^{m} x_{s}^{2}<\beta$; In addition, there exists a constant $\underline{D}>0$ such that $D_{i s}=D_{i s}(t, x, u) \geq \underline{D}>0$; denote $\underline{D}(m-2)^{2} / 2 \beta=x$, 
(2) $P_{i k}\left(u_{i}\left(t_{k}, x\right)\right)=-\theta_{i k} u_{i}\left(t_{k}, x\right), 1-\sqrt{1+\alpha} \leq \theta_{i k} \leq 1+\sqrt{1+\alpha}, \alpha \geq 0$,

(3) $\inf _{k=1,2, \ldots}\left(t_{k}-t_{k-1}\right)>\mu$,

(4) there exist constants $\gamma$ and $\varepsilon_{1}, \varepsilon_{2}>0$ such that $\gamma+\lambda+h \rho e^{\gamma \tau}>0$ and $\lambda+h \rho e^{\gamma \tau}+\ln (1+$ $\alpha) / \mu<0$, where $\lambda=\max _{i=1, \ldots, n}\left(-x-2 a_{i}+\sum_{j=1}^{n}\left(\varepsilon_{1} b_{i j}^{2}+\varepsilon_{2} c_{i j}^{2}\right)\right)+\left(n / \varepsilon_{1}\right) \max _{i=1, \ldots, n}\left(l_{i}^{2}\right)$ and $\rho=\left(n / \varepsilon_{2}\right) \max _{i=1, \ldots, n}\left(l_{i}^{2}\right)$.

Then, the equilibrium point $u=0$ of problem ((2.5)-(2.8)) is globally exponentially stable with convergence rate $-(1 / 2)\left(\lambda+h \rho e^{r \tau}+\ln (1+\alpha) / \mu\right)$.

Further, on the condition that $\left|P_{i k}\left(u_{i}\left(t_{k}, x\right)\right)\right| \leq \theta_{i k}\left|u_{i}\left(t_{k}, x\right)\right|$, where $\theta_{i k}^{2}<(\alpha-1) / 2$ and $\alpha \geq 1$, we obtain, for $t=t_{k}(k=1,2, \ldots)$,

$$
\begin{aligned}
u_{i}^{2}\left(t_{k}+0, x\right)-u_{i}^{2}\left(t_{k}, x\right) & =\left(P_{i k}\left(u_{i}\left(t_{k}, x\right)\right)+u_{i}\left(t_{k}, x\right)\right)^{2}-u_{i}^{2}\left(t_{k}, x\right) \\
& \leq 2\left(u_{i}\left(t_{k}, x\right)\right)^{2}+2\left(P_{i k}\left(u_{i}\left(t_{k}, x\right)\right)\right)^{2}-u_{i}^{2}\left(t_{k}, x\right) \\
& \leq\left(2+2 \theta_{i k}^{2}\right)\left(u_{i}\left(t_{k}, x\right)\right)^{2}-u_{i}^{2}\left(t_{k}, x\right) \leq \alpha u_{i}^{2}\left(t_{k}, x\right) .
\end{aligned}
$$

Identical with the proof of Theorem 3.3, we present the theorem as follows.

Theorem 3.7. Assume that

(1) for $x=\left(x_{1}, \ldots, x_{m}\right)^{T} \in \Omega(m \geq 3)$, there exists a constant $\beta$ such that $|x|^{2}=\sum_{s=1}^{m} x_{s}^{2}<\beta$. in addition, there exists a constant $\underline{D}>0$ such that $D_{i s}=D_{i s}(t, x, u) \geq \underline{D}>0$. Denote $\underline{D}(m-2)^{2} / 2 \beta=x$,

(2) $\left|P_{i k}\left(u_{i}\left(t_{k}, x\right)\right)\right| \leq \theta_{i k}\left|u_{i}\left(t_{k}, x\right)\right|$, where $\theta_{i k}^{2} \leq(\alpha-1) / 2$ and $\alpha \geq 1$,

(3) $\inf _{k=1,2, \ldots}\left(t_{k}-t_{k-1}\right)>\mu$,

(4) there exist constants $\gamma$ and $\varepsilon_{1}, \varepsilon_{2}>0$ such that $\gamma+\lambda+h \rho e^{\gamma \tau}>0$ and $\lambda+h \rho e^{\gamma \tau}+$ $\ln (1+\alpha) / \mu<0$, where $\lambda=\max _{i=1, \ldots, n}\left(-x-2 a_{i}+\sum_{j=1}^{n}\left(\varepsilon_{1} b^{2}{ }_{i j}+\varepsilon_{2} c_{i j}^{2}\right)\right)+$ $\left(n / \varepsilon_{1}\right) \max _{i=1, \ldots, n}\left(l_{i}^{2}\right)$ and $\rho=\left(n / \varepsilon_{2}\right) \max _{i=1, \ldots, n}\left(l_{i}^{2}\right)$.

Then, the equilibrium point $u=0$ of problem ((2.5)-(2.8)) is globally exponentially stable with convergence rate $-1 / 2\left(\lambda+h \rho e^{\gamma \tau}+\ln (1+\alpha) / \mu\right)$.

Remark 3.8. Different from Theorems 3.1-3.6, the impulsive part in Theorem 3.7 could be nonlinear and this will be of more applicability. Actually, Theorems 3.1-3.6 can be regarded as the special cases of Theorem 3.7.

\section{Examples}

Example 4.1. Consider the following impulsive reaction-diffusion delayed neural network:

$$
\begin{array}{r}
\frac{\partial u_{i}(t, x)}{\partial t}=\sum_{s=1}^{m} \frac{\partial}{\partial x_{s}}\left(D_{i s} \frac{\partial u_{i}(t, x)}{\partial x_{s}}\right)-a_{i} u_{i}(t, x)+\sum_{j=1}^{n} b_{i j} f_{j}\left(u_{j}(t, x)\right)+\sum_{j=1}^{n} c_{i j} f_{j}\left(u_{j}\left(t-\tau_{j}(t), x\right)\right) \\
t \geq 0, \quad t \neq t_{k}, \quad x \in \Omega, \quad k=1,2, \ldots, \quad i=1, \ldots, n
\end{array}
$$


with the impulsive effects characterized by

$$
\begin{array}{r}
u_{1}\left(t_{k}+0, x\right)=u_{1}\left(t_{k}, x\right)+1.343 u_{1}\left(t_{k}, x\right), \quad u_{2}\left(t_{k}+0, x\right)=u_{2}\left(t_{k}, x\right)+1.343 u_{2}\left(t_{k}, x\right) \\
k=1,2, \ldots, \quad x \in \Omega
\end{array}
$$

and initial condition (2.3) and Dirichlet condition (2.4), where $n=2, m=4, \Omega=$

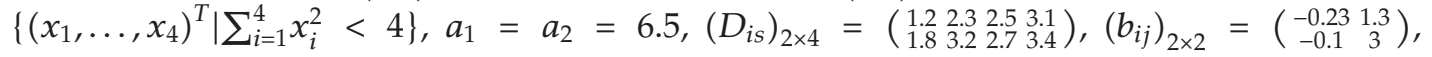
$\left(c_{i j}\right)_{2 \times 2}=\left(\begin{array}{c}-0.1-0.2 \\ 0.1\end{array}-0.3\right), f_{j}\left(u_{j}\right)=(1 / 4)\left(\left|u_{j}+1\right|-\left|u_{j}-1\right|\right), 0 \leq \tau_{j}(t) \leq 0.5$, and $\dot{\tau}_{j}(t)<0$. For $\beta=4$ and $\underline{D}=1.2$, we compute $\chi=0.6$. This, together with the chosen $l_{i}=1 / 2$, yields

$$
\mathcal{l}=\max _{i=1, \ldots, n}\left(-x-2 a_{i}+\sum_{j=1}^{n}\left(b_{i j}^{2}+c_{i j}^{2}\right)\right)+n \max _{i=1, \ldots, n}\left(l_{i}^{2}\right)=-4, \quad \rho=n \max _{i=1, \ldots, n}\left(l_{i}^{2}\right)=\frac{1}{2}
$$

By selecting $\gamma=2.6, \tau=0.5$ and $h=1$, we estimate

$$
\gamma+\lambda+h \rho e^{\gamma \tau}=2.6-4+\frac{1}{2} e^{1.3}>0, \quad \lambda+h \rho e^{\gamma \tau}=-4+\frac{1}{2} e^{1.3}<0 .
$$

According to Theorem 3.1, we therefore conclude that the system in Example 4.1 is globally exponential stable.

Example 4.2. Consider the following impulsive reaction-diffusion delayed neural network:

$$
\begin{array}{r}
\frac{\partial u_{i}(t, x)}{\partial t}=\sum_{s=1}^{m} \frac{\partial}{\partial x_{s}}\left(D_{i s} \frac{\partial u_{i}(t, x)}{\partial x_{s}}\right)-a_{i} u_{i}(t, x)+\sum_{j=1}^{n} b_{i j} f_{j}\left(u_{j}(t, x)\right)+\sum_{j=1}^{n} c_{i j} f_{j}\left(u_{j}\left(t-\tau_{j}(t), x\right)\right) \\
t \geq 0, \quad t \neq t_{k}, \quad x \in \Omega, \quad k=1,2, \ldots, \quad i=1, \ldots, n
\end{array}
$$

with the impulsive effects featured by

$$
\begin{array}{r}
u_{1}\left(t_{k}+0, x\right)=u_{1}\left(t_{k}, x\right)+\arctan \left(0.5 u_{1}\left(t_{k}, x\right)\right), \quad u_{2}\left(t_{k}+0, x\right)=u_{2}\left(t_{k}, x\right)+\arctan \left(0.5 u_{2}\left(t_{k}, x\right)\right) \\
k=1,2, \ldots, x \in \Omega
\end{array}
$$

and initial condition (2.3) and Dirichlet condition (2.4), where $n=2, m=4, \Omega=$

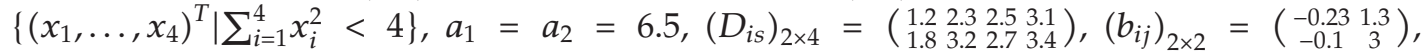
$\left(c_{i j}\right)_{2 \times 2}=\left(\begin{array}{cc}-0.1 & -0.2 \\ 0.1 & -0.3\end{array}\right), f_{j}\left(u_{j}\right)=1 / 4\left(\left|u_{j}+1\right|-\left|u_{j}-1\right|\right), 0 \leq \tau_{j}(t) \leq 0.5, \dot{\tau}_{j}(t)<0$, and 
$\inf _{k=1,2, \ldots}\left(t_{k}-t_{k-1}\right)>1$. For $\beta=4$ and $\underline{D}=1.2$, we compute $\mathcal{X}=0.6$. This, together with $l_{i}=1 / 2$ and $\varepsilon_{1}=\varepsilon_{2}=1$, yields

$$
\rho=\frac{n}{\varepsilon_{2}} \max _{i=1, \ldots, n}\left(l_{i}^{2}\right)=\frac{1}{2}, \quad \lambda=\max _{i=1, \ldots, n}\left(-x-2 a_{i}+\sum_{j=1}^{n}\left(\varepsilon_{1} b_{i j}^{2}+\varepsilon_{2} c_{i j}^{2}\right)\right)+\frac{n}{\varepsilon_{1}} \max _{i=1, \ldots, n}\left(l_{i}^{2}\right)=-4 .
$$

Select $\alpha=1.5$ by setting $\theta_{i k}=0.5$. Hence, we compute by letting $\mu=1, \gamma=3, \tau=0.5$, and $h=1$ that

$$
\gamma+\lambda+h \rho e^{\gamma \tau}=3-4+\frac{1}{2} e^{1.5}>0, \quad \lambda+h \rho e^{\gamma \tau}+\frac{\ln (1+\alpha)}{\mu}=-4+\frac{1}{2} e^{1.5}+\ln 2.5<0 .
$$

It is then concluded from Theorem 3.7 that the system in Example 4.2 is globally exponentially stable.

\section{Acknowledgment}

The work is supported by National Natural Science Foundation of China under Grant 60904028.

\section{References}

[1] L. O. Chua and L. Yang, "Cellular neural networks: theory," IEEE Transactions on Circuits and Systems, vol. 35, no. 10, pp. 1257-1272, 1988.

[2] L. O. Chua and L. Yang, "Cellular neural networks: applications," IEEE Transactions on Circuits and Systems, vol. 35, no. 10, pp. 1273-1290, 1988.

[3] J. Cao, "New results concerning exponential stability and periodic solutions of delayed cellular neural networks," Physics Letters A, vol. 307, no. 2-3, pp. 136-147, 2003.

[4] J. Cao, "On stability of cellular neural networks with delay," IEEE Transactions on Circuits and Systems, vol. 40, no. 3, pp. 157-165, 1993.

[5] P. P. Civalleri and M. Gilli, "A set of stability criteria for delayed cellular neural networks," IEEE Transactions on Circuits and Systems. I, vol. 48, no. 4, pp. 494-498, 2001.

[6] J. J. Hopfield, "Neurons with graded response have collective computational properties like those of two-state neurons," Proceedings of the National Academy of Sciences of the United States of America, vol. 81, no. 10 I, pp. 3088-3092, 1984.

[7] J. Yan and J. Shen, "Impulsive stabilization of functional-differential equations by LyapunovRazumikhin functions," Nonlinear Analysis. Theory, Methods \& Applications, vol. 37, no. 2, pp. 245-255, 1999.

[8] X. Liu and Q. Wang, "Impulsive stabilization of high-order Hopfield-type neural networks with timevarying delays," IEEE Transactions on Neural Networks, vol. 19, no. 1, pp. 71-79, 2008.

[9] X. Liu, "Stability results for impulsive differential systems with applications to population growth models," Dynamics and Stability of Systems, vol. 9, no. 2, pp. 163-174, 1994.

[10] S. Arik and V. Tavsanoglu, "On the global asymptotic stability of delayed cellular neural networks," IEEE Transactions on Circuits and Systems. I, vol. 47, no. 4, pp. 571-574, 2000.

[11] L. O. Chua and T. Roska, "Stability of a class of nonreciprocal cellular neural networks," IEEE Transactions on Circuits and Systems, vol. 37, no. 12, pp. 1520-1527, 1990.

[12] Z.-H. Guan and G. Chen, "On delayed impulsive Hopfield neural networks," Neural Networks, vol. 12, no. 2, pp. 273-280, 1999.

[13] Q. Zhang, X. Wei, and J. Xu, "On global exponential stability of delayed cellular neural networks with time-varying delays," Applied Mathematics and Computation, vol. 162, no. 2, pp. 679-686, 2005. 
[14] D. D. Bainov and P. S. Simeonov, Systems with Impulse Effect, Ellis Horwood Ltd., Chichester, UK, 1989.

[15] I. M. Stamova, Stability Analysis of Impulsive Functional Differential Equations, Walter de Gruyter, Berlin, Germany, 2009.

[16] M. A. Arbib, Brains, Machines, and Mathematics, Springer, New York, NY, USA, 2nd edition, 1987.

[17] S. Haykin, Neural Networks: A Comprehensive Foundation, Prentice-Hall, Englewood Cliffs, NJ, USA, 1998.

[18] H. Akça, R. Alassar, V. Covachev, Z. Covacheva, and E. Al-Zahrani, "Continuous-time additive Hopfield-type neural networks with impulses," Journal of Mathematical Analysis and Applications, vol. 290, no. 2, pp. 436-451, 2004.

[19] G. T. Stamov, "Almost periodic models of impulsive Hopfield neural networks," Journal of Mathematics of Kyoto University, vol. 49, no. 1, pp. 57-67, 2009.

[20] G. T. Stamov and I. M. Stamova, "Almost periodic solutions for impulsive neural networks with delay," Applied Mathematical Modelling, vol. 31, no. 7, pp. 1263-1270, 2007.

[21] S. Ahmad and I. M. Stamova, "Global exponential stability for impulsive cellular neural networks with time-varying delays," Nonlinear Analysis. Theory, Methods \& Applications, vol. 69, no. 3, pp. 786795, 2008.

[22] X. Liu, K. L. Teo, and B. Xu, “Exponential stability of impulsive high-order Hopfield-type neural networks with time-varying delays," IEEE Transactions on Neural Networks, vol. 16, no. 6, pp. 13291339, 2005.

[23] J. Qiu, "Exponential stability of impulsive neural networks with time-varying delays and reactiondiffusion terms," Neurocomputing, vol. 70, no. 4-6, pp. 1102-1108, 2007.

[24] K. Li and Q. Song, "Exponential stability of impulsive Cohen-Grossberg neural networks with timevarying delays and reaction-diffusion terms," Neurocomputing, vol. 72, no. 1-3, pp. 231-240, 2008.

[25] J. Pan, X. Liu, and S. Zhong, "Stability criteria for impulsive reaction-diffusion Cohen-Grossberg neural networks with time-varying delays," Mathematical and Computer Modelling, vol. 51, no. 9-10, pp. 1037-1050, 2010.

[26] V. Lakshmikantham, D. D. Bă̌nov, and P. S. Simeonov, Theory of Impulsive Differential Equations, vol. 6, World Scientific, Teaneck, NJ, USA, 1989.

[27] Adimurthi, "Hardy-Sobolev inequality in $H^{1}(\Omega)$ and its applications," Communications in Contemporary Mathematics, vol. 4, no. 3, pp. 409-434, 2002. 


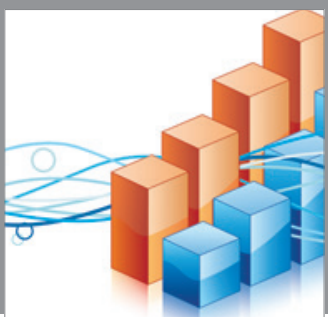

Advances in

Operations Research

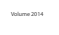

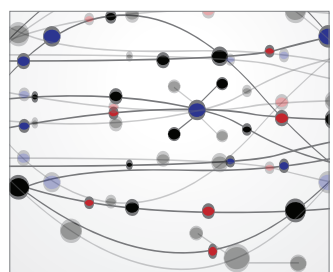

\section{The Scientific} World Journal
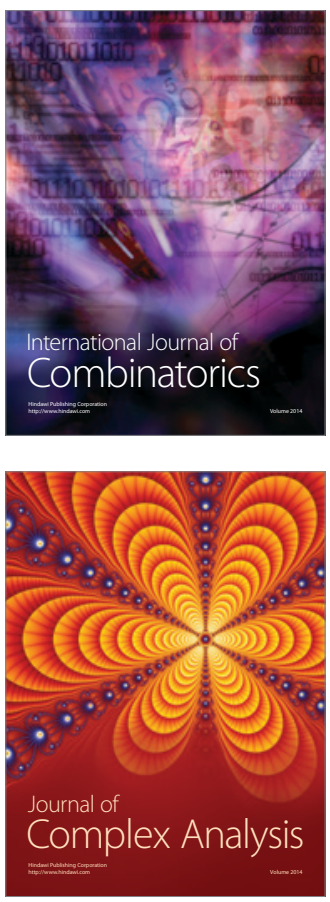

International Journal of

Mathematics and

Mathematical

Sciences
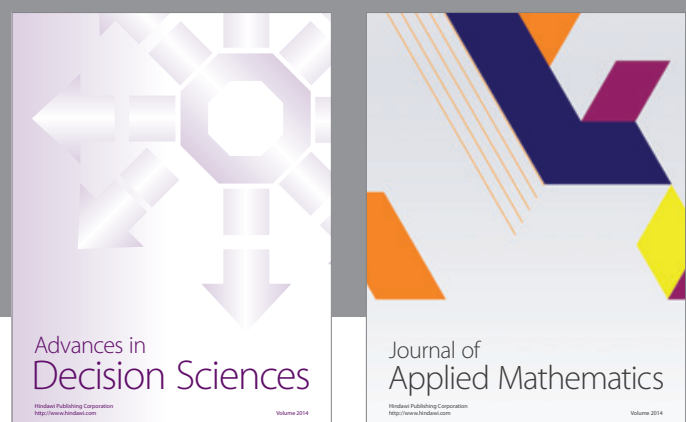

Journal of

Applied Mathematics
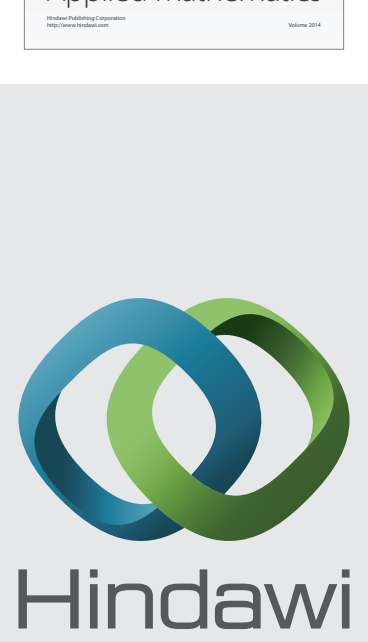

Submit your manuscripts at http://www.hindawi.com
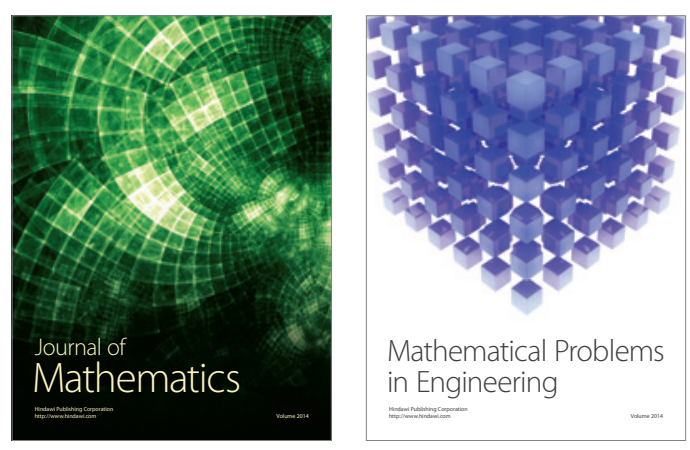

Mathematical Problems in Engineering
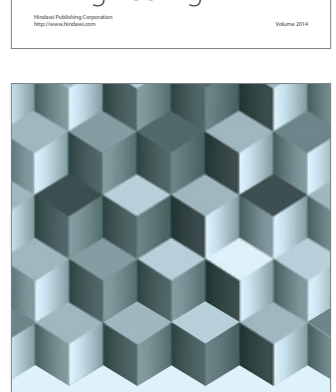

Journal of

Function Spaces
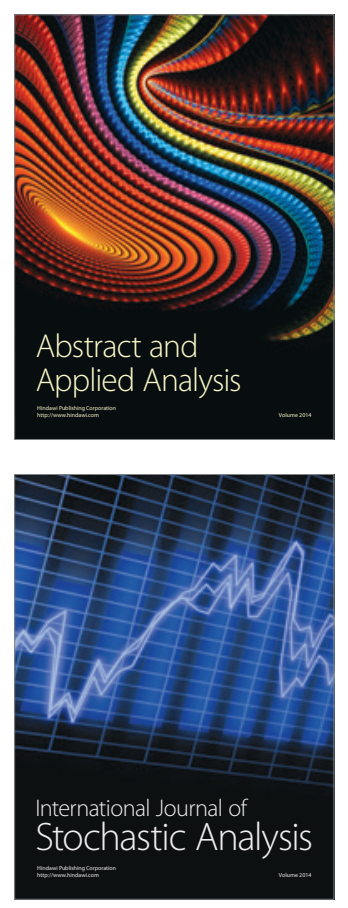

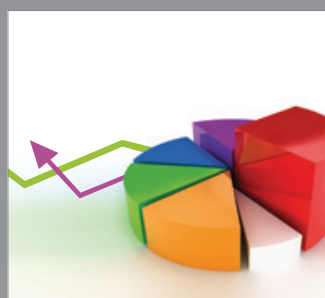

ournal of

Probability and Statistics

Promensencen
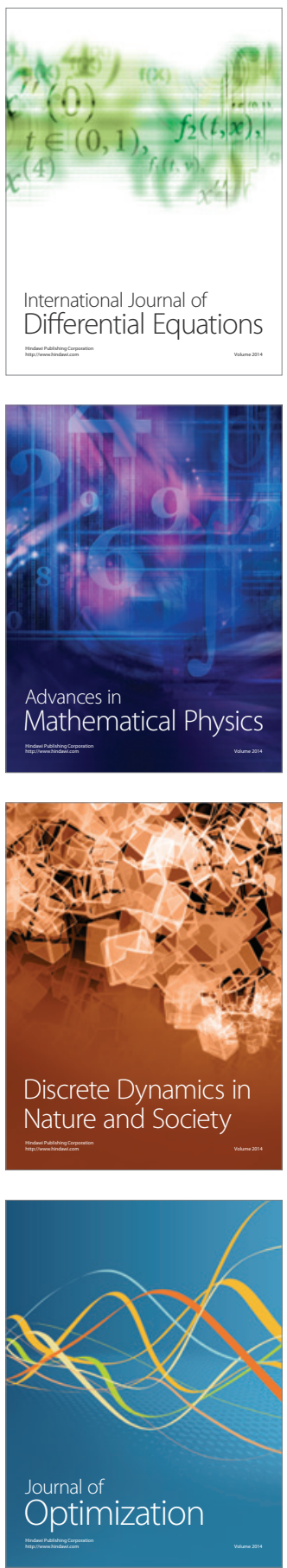